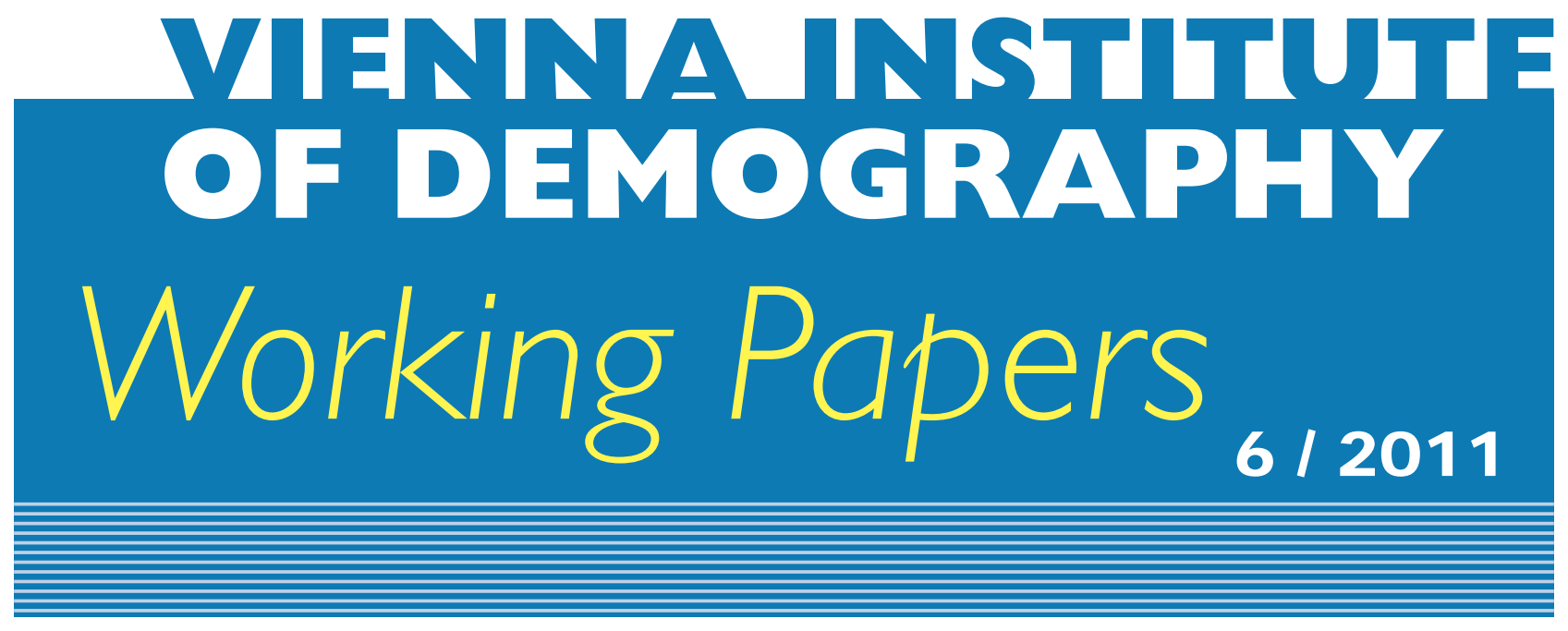

Gustav Feichtinger, M ichael Kuhn, Alexia Prskawetz, and Stefan W rzaczek

\title{
The Reproductive Value as Part of the Shadow Price of Population
}

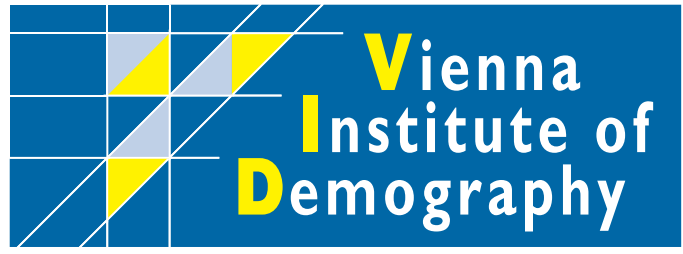

Vienna Institute of Demography Austrian Academy of Sciences

Wohllebengasse $12-14$

A-I040Vienna $\cdot$ Austria

E-Mail:vid@oeaw.ac.at

Website: www.oeaw.ac.at/vid 


\begin{abstract}
The reproductive value (see Fisher 1930) arises as part of the shadow price of the population in a large class of age-structured optimal control models.
\end{abstract}

\title{
Keywords
}

Reproductive value, distributed optimal control theory, McKendrick equation, shadow price, indirect effect.

\section{Authors}

Gustav Feichtinger, Vienna University of Technology, Institute of Mathematical Methods in Economics, research group on Operations Research and Control Systems.

Email: gustav@server.eos.tuwien.ac.at

Michael Kuhn, Austrian Academy of Sciences, Vienna Institute of Demography.

Email: michael.kuhn@oeaw.ac.at

Alexia Prskawetz, Vienna University of Technology, Institute of Mathematical Methods in Economics, research group on Economics, and Austrian Academy of Sciences, Vienna Institute of Demography. Email: afp@econ.tuwien.ac.at

Stefan Wrzaczek, Vienna University of Technology, Institute of Mathematical Methods in Economics, research group on Operations Research and Control Systems, and Austrian Academy of Sciences, Vienna Institute of Demography.

Email:wrzaczek@server.eos.tuwien.ac.at

\section{Acknowledgement}

This research was partly financed by the European Commission under grant SSH-20073.1.01- 217275 (Long-Run Economic Perspectives of Ageing Societies). 


\title{
The Reproductive Value as Part of the Shadow Price of Population
}

\author{
Gustav Feichtinger, Michael Kuhn, Alexia Prskawetz, Stefan Wrzaczek
}

\section{Relationship}

The reproductive value at age $a$, introduced by Fisher [16], is defined as

$$
v(a)=\int_{a}^{\beta} e^{-r(s-a)} \frac{l(s)}{l(a)} m(s) d s,
$$

where $l(s)$ denotes the probability to survive from birth until age $s, m(s)$ the fertility rate of age $s, \beta$ the oldest age of childbearing and $r$ the discount rate, which is equal to Lotka's $r$ (see e.g. Keyfitz [22]).

Consider an optimal control model in which an intertemporal objective functional is to be optimised over a finite time horizon, where the objective itself depends on the size and age-structure of some population. Further assume that within such an age-structured optimal control model, population aged $a$ at time $t$ is used as a state variable $N(a, t)$, described by the McKendrick equation (see Keyfitz [22] and Keyfitz and Keyfitz [23]), then the shadow price attached to population $\xi^{N}(a, t)$ typically embraces a generalization of the reproductive value. $\xi^{N}(a, t)$ itself can be interpreted as the marginal value of an additional $a$-year old individual at time $t$. The relationships become clearer when considering the following decomposition ${ }^{1}$

$$
\xi^{N}(a, t)=\xi^{d}(a, t)+\int_{a}^{\omega} e^{-\rho(s-a)} \frac{l(s, \cdot)}{l(a, \cdot)} m(s, \cdot) \xi^{N}(0, t-a+s) d s,
$$

for $t-a+\omega<T$, where $\omega$ denotes the maximal length of life, $t$ time, $a$ age and $\rho$ the time discount rate. The direct effect $\xi^{d}(a, t)$ accounts for the marginal value of the population that is currently alive. The indirect effect, as given by the second term on the right hand side, accounts for the marginal value of expected newborns and is a generalized form of the classical reproductive value (1). Put differently the indirect effect could represent the economic value of the lineage associated with an additional individual.

Note that the integral in the above result is a variant of Fisher's formulation. The integrand, the discounted expected number of newborns at time $t-a+s$, is weighted by their value to the decision-maker, as given by the shadow price $\xi^{N}(0, t-a+s)$. In Fisher's case this value equals one, since he regarded the birth of a child as lending to him of a life. Moreover, while the discount rate is equal to Lotka's $r$ in (1), in our

\footnotetext{
${ }^{1}$ Equation (2) is derived in section 2 (see equations (4)-(6)).
} 
expression (2) the discount rate is equal to the time preference $\rho$ and exogenously given. Note that it makes no difference that the upper bound of the integral is the maximal length of life, since the fertility rate is zero after the maximal age of childbearing.

The generality of the above expression has two important implications. Firstly, in Fisher's original formulation (1) the population is stable. The above expression allows for changes in the demography over time. ${ }^{2}$ Secondly, the sign of the reproductive value is always positive. Due to the weighting with $\xi^{N}(0, t-a+s)$, the general expression can also have a negative sign.

\section{Proof}

Consider the following age-structured optimal control model ${ }^{3,4}$

$$
\begin{aligned}
V(N(a, 0))=\max _{u \in U} & \int_{0}^{T} \int_{0}^{\omega} e^{-\rho t} L(a, t, N, u) d a d t \\
\text { s.t. } \quad\left(\frac{\partial}{\partial a}+\frac{\partial}{\partial t}\right) N(a, t) & =-\mu(a, t, u) N(a, t) \\
N(0, t) & =B(t)=\int_{0}^{\omega} m(a, t, u) N(a, t) d a, N(a, 0)=N_{0}(a)
\end{aligned}
$$

where population dynamics is modeled according to the McKendrick equation with an endogenous number of newborns $B(t)$. The decision maker chooses the control $u(a, t)$ from the set of admissible controls $U$ such that the objective, equal to the discounted instantaneous objective functionals ${ }^{5} L(\cdot)$ aggregated over age and time, is maximized over a finite time horizon $T<\infty$ with a zero salvage value. The control $u(a, t)$ itself influences the objective functionals as well as the mortality and the fertility rates.

For age-structured optimal control models with additional cohort states (45-degree line in the Lexis diagram), period states (90-degree line in the Lexis diagramm) and a non-zero salvage value we refer to Wrzaczek et al. [31].

According to the Maximum principle for age-structured optimal control models (see Brokate [4] or Feichtinger et al. [14] $)^{6}$ we formulate the current-value Hamiltonian,

\footnotetext{
${ }^{2}$ See also Ediev[10] on the dynamics of the reproductive value.

${ }^{3}$ For simplification $a$ and $t$ are omitted sometimes.

${ }^{4} V(N(a, 0))$ denotes the optimal value of the objective functional, which depends on $N(a, 0)$. In dynamic optimisation $V(N(a, t))$ is referred to as value function.

${ }^{5}$ For instance, the aggregate objective could be intertemporal social welfare. In this case, the objective functional $L(\cdot)$ would measure the instantaneous welfare of age-group $N(a, t)$. In different settings, $L(\cdot)$ may describe the (economic) value ascribed to an animal or plant population.

${ }^{6}$ The Maximum Principle presented in Feichtinger et al. [14] is a generalization of that in Brokate [4]. Firstly the formulation of the model allows for more general forms of the objective function and the dynamics. Secondly, an additional type of state accounting for interactions between the cohorts (often important in epidemiological models) is allowed. On the other hand, the Maximum Principle of
} 
which consists of the instantaneous objective functional (current contribution) and the dynamics (population as well as the integral constraint ${ }^{7}$ ) weighted by the adjoint variable (future contribution), i.e.

$$
\mathcal{H}=L(a, t, N, u)-\xi^{N}(a, t) \mu(a, t, u) N(a, t)+\xi^{N}(0, t) m(a, t, u) N(a, t)
$$

and maximize it with respect to the control $u(a, t)$. The Hamiltonian may be seen as a generalization of the Lagrangean method (for an excellent reference see Leonard and van Long [26]). We further derive the adjoint equation equal to the negative partial derivative of the Hamiltonian with respect to population $N(a, t)$ plus a term due to discounting the future, i.e.

$$
\left(\frac{\partial}{\partial a}+\frac{\partial}{\partial t}\right) \xi^{N}(a, t)=(\rho+\mu) \xi^{N}(\cdot)-\frac{\partial L(\cdot)}{\partial N}-\xi^{N}(0, t-a+s) m(\cdot)
$$

In this expression the optimal value of the control has already been used. Together with the corresponding transversality condition $\xi^{N}(\omega, t)=0$ the above differential equation can be turned into an equivalent integral equation, which can be solved recursively. We obtain $^{8}$

$$
\begin{aligned}
\xi^{N}(a, t)= & \int_{a}^{\omega} e^{-\rho(s-a)} \frac{l(s, t-a+s)}{l(a, t)} \frac{\partial L(\cdot)}{\partial N} d s+ \\
& +\int_{a}^{\omega} e^{-\rho(s-a)} \frac{l(s, t-a+s)}{l(a, t)} m(s, t-a+s, u(\cdot)) \xi^{N}(0, t-a+s) d s .(6)
\end{aligned}
$$

The direct effect is represented by the first integral. It is equal to the marginal effect of the population on the instantaneous objective functional $\frac{\partial L(\cdot)}{\partial N}$. Discounted by $\rho$ and weighted by the survival probability, this effect is aggregated over the remaining life of the cohort born at $t-a$. The second integral is the more general form of Fisher's reproductive value as discussed in the previous section.

For a detailed discussion on a general model and mathematical details (assumptions on the functions involved, conditions for the existence of solutions) we refer to Wrzaczek et al. [31] and Feichtinger et al. [14]. For a model with a male and female population the analysis is analogous and the core result does not change.

Brokate [4] allows for an infinite life-time horizon, which is not contained in that of Feichtinger et al. [14]

${ }^{7}$ The integrand of $B(t)$ is weighted by a separate adjoint variable $\eta(t)$. However, since $B(t)$ only acts as boundary constraint of the population $\eta(t)=\xi^{N}(0, t)$ follows immediately.

${ }^{8}$ Note that the conditional survival probability $\frac{l(s, t-a+s)}{l(a, t)}$ is a transformation of the term $e^{-\int_{a}^{s} \mu(\cdot) d s^{\prime}}$, which originally enters according to the adjoint equation $(5)$. For greater clearness we have chosen the demographic notation. 


\section{$3 \quad$ History and related results}

While intertemporal optimization is ubiquitous in population economics, ${ }^{9}$ it is not a standard tool in demography. One main purpose of the present note is to illustrate its applicability in population dynamics. Since population processes evolve in time we use intertemporal optimization in particular optimal control theory. Its main advantage is to provide qualitative insights into structural properties of optimal solution paths. The core concept of dynamic optimization is the shadow price of the state variable. It measures the marginal value (in terms of the objective function) of an increment of the state at time $t$ when moving along the optimal path. Assume that the decision maker who is faced with a state $N(a, t)$ at time $t$ decides optimally from $t$ onwards until the end of the process. The shadow price $\xi^{N}(a, t)$ is defined as the partial derivative of the optimal value of the process $V(\cdot)$ with respect to $N(a, t)$, i.e.

$$
\xi^{N}(a, t)=\frac{\partial V(N(a, t))}{\partial N(a, t)}
$$

This imputed (marginal) value can be seen as a dynamic extension of the dual variable in (static) mathematical (linear and non-linear) programming. Note that it is no market price (which must be always non-negative), but measures the marginal impact of an additional (infitesimal) unit of the state on the objective. In optimal control theory this dynamic shadow price is referred to as adjoint variable. Clearly, shadow prices can take negative values.

At any instant of time $t$, the decision-maker considers the state of the system $N(a, t)$ and has to choose the control $u(a, t)$ optimally. This selection has two effects (see e.g. Grass et al. [18]). (i) to generate an instantaneous utility $L(a, t, N, u$ ), and (ii) to change the state through the system dynamics, i.e. in our case $-\mu(a, t, u) N(a, t)$ as well as the boundary constraint $B(t)$. Thus, a rational decision maker has to take into consideration the change of the state appropriately.

Our indirect effect that shows up in the dynamics of the shadow price (equation (2)) may therefore be seen as a further alternative of the valuation of the reproductive value at age zero, by taking into account the marginal value of a newborn as given by the shadow price at age zero.

Life-history theories of ageing aim for an explanation of the genetic architecture of the life-history. Life-histories are defined as cycles of maturation, fertility and mortality (see e.g. Charlesworth [6]). Remarkably, there is an interesting analogy with a finding by Goodman [17] who considers an optimal control model to deal with life-history optimisation $^{10}$, where a decision-maker (evolution) chooses optimal trajectories of fertility (rates) in order to maximise the total reproductive contribution of cohort at birth subject to certain physiological constraint. He shows that the reproductive value (at age

\footnotetext{
${ }^{9}$ For an early example we refer to Arthur and McNicoll [1].

${ }^{10}$ For an even earlier dynamic programming and optimal control approach to life history optimisation see Leon [25] or Taylor et al. [30].
} 
$a$ ) is the current shadow price of survival of an age $a$ individual in the cohort. Thus, for an optimal life-history model any individual is valued exactly at its reproductive value, whereas in our model, in which population may have a more general value than reproduction alone, the weighted reproductive value constitutes one part of an individual's value.

Notably, the shadow price of population bears close resemblance to the economicdemographic potential (see Ediev [7], [8], [9] based on earlier measures as e.g. by Hersch [19] and Burgeois-Pichat [5]). While the demographic potential measures a population's prospects for future growth (Ediev [7], [8]), the economic-demographic potential extends this to provide an index reflecting the net present value of future economic flows originating from both the present generation (our direct effect $\xi^{d}(a, t)$ ) and future generations (our indirect effect). By deriving the shadow price of population within an explicit optimal control problem, our approach lends a foundation to the economic-demographic potential as measure of an optimal 'policy'. Indeed, by its very nature the shadow price embraces a measure of the future economic-demographic prospects.

In a similar vein, economists have thought since long about the value of (human) life. Hofflander [20] provides an excellent survey of such thought, including prominent contributions by William Farr and Alfred Marshall. Schelling [28] was first to (re-)introduce the concept in a modern way, which was subsequently formalized by Shepard and Zeckhauser [29]. The value of a statistical life, rigorously defined as the (hypothetical) monetary value an individual would attach to its survival from an exante perspective, is typically derived within a life-cycle model, where an individual maxmises utility over the remaining (expected) life course, but does not account for its progeny. For such an egoistic individual the value of life only depends on the prospect over the own remaining life course, corresponding to the direct term $\xi^{d}(a, t)$ in expression (2). Recent work by Birchenall and Soares [3] and by Kuhn et al. [24] accounts for the presence of altruism towards descendants in the spirit of Becker and Barro [2]. In this case the value of an individual's life is amended by the value the individual attaches to its prog eny (depending inter alia on their future prospects), corresponding to the indirect effect in (2). In practical terms this implies that the value of life may well be underestimated for individuals who are still within their fertile years.

\section{Applications}

In section 1 we mentioned that the generalized reproductive value can have a negative sign. As an example consider a model minimizing the impact of a population of a pest on a valuable store of a resource. If the pest population (destroying the resource) is modeled in the above way, the corresponding shadow price will be negative. This implies that also the generalized reproductive value (i.e. the impact of yet to be born pest individuals on the future stock of the resource) is negative.

For another application consider an age-specific predator-prey model, embracing 
cattle and wolves, say, as presented in Wrzaczek et al. [31]. In this model the dynamics of the predator and prey population is modeled by a generalized form of the McKendrick equation in order to account for the interaction between them. The more prey is alive the better is the nutrition for the predator, and the more predators are alive the more prey will be killed. Using these dynamics when optimizing a general objective function (e.g. the net economic value of the cattle population) leads to an interesting interactio of the shadow prices relating to the two populations. In addition to the generalized reproductive vlaue, the shadow price of the prey enters the direct effect of the predator and vice versa. This accounts for the fact that the prey population represent the

predator's food supply and the predator population represents a prime cause for prey mortality. Also in this example the reproductive term can be negative depending on the type of objective function (e.g. the reproductive vlaue of the predator if in contrast to the prey it has no direct economic value in and of itself).

A further epidemiological application involving the interaction of infected and susceptible individuals is presented in Wrzaczek et al. [31].

Finally, we want to emphasize that the concept of the reproductive value is not only applicable for humans and animals, but also for self-renewable machines or even capital (for examples see e.g. Sethi and Thompson [27]). In several models machines have vintage structure in order to account for technological progress (see e.g. Feichtinger et al. [12] and references therein). If then new machines are produced by using existing machines, the corresponding shadow price has the same structure as discussed above. In that case the birth rate can be interpreted as productivity of existing machines producing new ones. Due to technical progress the productivity will increase over time. The survival probability equals the value of the machines according to physical depreciation.

\section{References}

[1] Arthur, W.B., McNicoll, G. (1977) Optimal Time Paths with Age-Dependence: A Theory of Population Policy, Review of Economic Studies 44 (1), 111-123.

[2] Becker, G.S., Barro, R.J. (1988). A reformulation of the economic theory of fertility, Quarterly Journal of Economics 103, 1-25.

[3] Birchenall, J.A., Soares, R.R. (2009). Altruism, fertility, and the value of children: Health policy evaluation and intergenerational welfare. Journal of Public Economics 93, 280-295.

[4] Brokate, M. (1985). Pontryagin's principle for control problems in age-dependent population dynamics, Journal of Mathematical Biology 23, 75-101.

[5] Burgeois-Pichat, J. (1951) Les limites de la demographie potentielle. Review of the International Statistical Institute 19, 13-27. 
[6] Charlesworth, B. (1994). Evolution in age-structured populations. Cambridge University Press, second edition.

[7] Ediev D.M. (2001) Application of the Demographic Potential Concept To Understanding the Russian Population History and Prospects: 1897-2100. Demographic Research 4, 289-336.

[8] Ediev D.M. (1996) Economic Analysis of Demographic Dynamics. Modeling the Processes of Management and Information Processing. Moscow: Moscow Institute of Physics and Technology, 76-80.

[9] Ediev D.M. (2007a) Demographic potentials: theory and applications. Moscow: Max-Press.

[10] Ediev, D.M. (2007b). On an extension of R. A. Fisher's result on the dynamics of the reproductive value. Theoretical Population Biology 72, 480-484.

[11] Farr, W. (1853) The income and property tax. Quaterly Journal of the Statistical Society 16, 4.

[12] Feichtinger, G., Kort, P.M., Hartl, R.F., Veliov, V.M. (2006). Anticipation effects of technological progress on capital accumulation: a vintage capital approach. Journal of Economic Theory 126, 143-164.

[13] Feichtinger, G., Prskawetz, A., Veliov, V.M. (2994) Age structured optimal control in population economics. Theoretical Population Biology 65, 373-387.

[14] Feichtinger, G., Tragler, G., Veliov, V.M. (2003). Optimality conditions for agestructured control systems. Journal of Mathematical Analysis and Applications $288,47-68$.

[15] Fisher, R.A. (1927). The actuarial treatment of official birth records, Eugenics Review, 19, 103-108.

[16] Fisher, R.A. (1930). The genetical theory of natural selection. Dover Publications, New York.

[17] Goodman, D. (1982) Optimal life histories, optimal notation, and the value of reproductive value. American Naturalist 119, 803-823.

[18] Grass, D., Calkins, J.P., Feichtinger, G., Tragler, G., Behrens, D. (2008) Optimal control of nonlinear processes: With applications in drugs, corruption and terror. Springer, Berlin.

[19] Hersch, L. (1944) Quelques precisions sur la methode des potentiels-vie et ses notions fondamentales. Review of the International Statistical Institute 12, 23-35. 
[20] Hofflander, A.E. (1966) The Human Life Value: An Historical Perspective. Journal of Risk and Insurance 33 (3), 381-391.

[21] Keyfitz, N. (1971). On the momentum of population growth. Demography, 8, 71-80.

[22] Keyfitz, N. (1977). Applied mathematical demography. John Wiley \& Sons.

[23] Keyfitz, B.L., Keyfitz, N. (1997). The McKendrick partial differential equation and its uses in epidemiology and population study. Mathematical and Computer Modelling 26 (6), 1-9.

[24] Kuhn, M., Wrzaczek, S., Oeppen, J. (2010). Recognizing progeny in the value of life. Economics Letters 107, 17-21.

[25] Leon, J.A. (1976). Life histories as adaptive strategies. Journal of theoretical Biology 60, 301-335.

[26] Leonard, D., Van Long, N. (1992). Optimal Control Theory and Static Optimization in Economics. Cambridge University Press.

[27] Sethi, S.P., Thompson, G.L. (2000). Optimal control theory. Applications to management science and economics. Kluwer Academic Publishers.

[28] Schelling, T.C. (1968). The life you save may be your own. In: Chase, S.B. Problems in public expenditures analysis. The Brooking Institution, Washington D.C.

[29] Shepard, D.S., Zeckhauser, R.J. (1982). Life-cycle consumption and willingness to pay for increased survival. In: Jones-Lee, M.N.: The Value of Life and Safety, North-Holland Publishing Company.

[30] Taylor, H.M., Gourley, R.S., Lawrence, C.E., Kaplan, R.S. (1974) Natural Selection of Life History Attributes: An Analytical Approach. Theoretical Population Biology 5, 104-122.

[31] Wrzaczek, S., Kuhn, M., Prskawetz, A., Feichtinger, G. (2010). The Reproductive value in distributed optimal control models. Theoretical Population Biology 77, 164-170. 


\section{VIENNA INSTITUTE OF DEMOGRAPHY}

\section{Working Papers}

Barakat, Bilal, Time is Money: Could Deferred Graduate Retirement Finance Higher Education?, VID Working Paper 05/2011.

Riosmena, Fernando, Maria Winkler-Dworak, Alexia Prskawetz, and Gustav Feichtinger, The Impact of Policies Influencing the Demography of Age Structured Populations: Lessons from Academies of Science, VID Working Paper 04/2011.

Buber, Isabella and Katrin Fliegenschnee, Are you Ready for a Child? A Methodological Triangulation on Fertility Intentions in Austria, VID Working Paper $03 / 2011$.

Fent, Thomas, Belinda Aparicio Diaz, and Alexia Prskawetz, Family Policies in the Context of Low Fertility and Social Structure, VID Working Paper 02/2011.

Buber, Isabella, Caroline Berghammer, and Alexia Prskawetz, Doing Science, Forgoing Childbearing? Evidence from a Sample of Female Scientists in Austria, VID Working Paper 01/2011.

Strulik, Holger, Klaus Prettner, and Alexia Prskawetz, R\&D-Based Growth in the Post-Modern Era, VID Working Paper 09/2010.

Prettner, Klaus and Alexia Prskawetz, Demographic Change in Models of Endogenous Economic Growth. A Survey, VID Working Paper 08/2010.

Flandorfer, Priska, Christian Wegner, and Isabella Buber, Gender Roles and Smoking Behaviour, VID Working Paper 07/2010.

Barakat, Bilal, Johannes Holler, Klaus Prettner, and Julia Schuster, The Impact of the Economic Crisis on Labour and Education in Europe, VID Working Paper 06/2010.

Grafeneder-Weissteiner, Theresa and Klaus Prettner, Agglomeration Processes in Ageing Societies, VID Working Paper 05/2010.

Buber, Isabella, Parity-specific weights for the Austrian Generations and Gender Survey, VID Working Paper 04/2010.

Testa, Maria Rita, She wants, he wants: Couple's childbearing desires in Austria, VID Working Paper 03/2010.

The Vienna Institute of Demography Working Paper Series receives only limited review. Views or opinions expressed herein are entirely those of the authors. 